\title{
New Results on Global Exponential Stability of Impulsive Functional Differential Systems with Delayed Impulses
}

\author{
Pei Cheng, Zheng Wu, and Lianglong Wang \\ School of Mathematical Sciences, Anhui University, Hefei 230601, China \\ Correspondence should be addressed to Lianglong Wang, wangll@ahu.edu.cn
}

Received 20 June 2012; Accepted 7 August 2012

Academic Editor: Elena Braverman

Copyright (c) 2012 Pei Cheng et al. This is an open access article distributed under the Creative Commons Attribution License, which permits unrestricted use, distribution, and reproduction in any medium, provided the original work is properly cited.

By using the Lyapunov functions and the Razumikhin techniques, the exponential stability of impulsive functional differential systems with delayed impulses is investigated. The obtained results have shown that the system will stable if the impulses' frequency and amplitude are suitably related to the increase or decrease of the continuous flows, and they improve and complement ones from some recent works. An example is provided to illustrate the effectiveness and the advantages of the results obtained.

\section{Introduction}

There has been a growing interest in the theory of impulsive dynamical systems in the past decades because of their applications to various problems arising in communications, control technology, impact mechanics, electrical engineering, medicine, biology and so forth; see the monographs $[1,2]$ and the papers [3-8] and the references therein. In particular, special attention has been focused on stability and impulsive stabilization of impulsive functional differential systems (IFDSs) (see, e.g., [9-26]).

However, in these previous works on stability of IFDSs, the authors always suppose that the state variables on the impulses are only related to the present state variables. But in most cases, it is more applicable that the state variables on the impulses that we add are also related to the past ones. For example, it is more realistic in practice if the impulsive control depends on a past state due to a time lag between the time when the observation of the state is made and the time when the feedback control reaches the system.

In fact, there have been several attempts in the literature to study the stability and control problems of a particular class of IFDSs with delayed impulses (see, e.g., [27-36]). 
Lian et al. [27] investigated the optimal control problem of linear continuous-time systems possessing delayed discrete-time controllers in networked control systems. For nonlinear impulsive systems, Khadra et al. studied the impulsive synchronization problem coupled by linear delayed impulses in [28]. In addition, in [29-34], the authors investigate the uniform asymptotic stability and global exponential stability of general IFDSs:

$$
\begin{aligned}
& \dot{x}(t)=f\left(t, x_{t}\right), \quad t \neq t_{k}, \quad t \geqslant t_{0}, \\
& \Delta x\left(t_{k}\right)=I_{k}\left(t_{k}, x_{t_{k}^{-}}\right), \quad k \in \mathbb{Z}_{+} .
\end{aligned}
$$

But in these stability analyses, the effects of time delay on the impulses have been ignored. For example in [31-34], the Lyapunov function was assumed to be satisfied $V\left(t_{k}, \varphi(0)+\right.$ $\left.I_{k}\left(t_{k}, \varphi\right)\right) \leqslant\left(1+d_{k}\right) V\left(t_{k}^{-}, \varphi(0)\right)$.

Very recently, in [35], Zhang and Sun established some sufficient conditions for uniform stability, uniform asymptotical stability, and practical stability of a particular class of IFDSs with delayed impulses:

$$
\begin{gathered}
\dot{x}(t)=f\left(t, x_{t}\right), \quad t \neq t_{k}, t \geqslant t_{0}, \\
\Delta x\left(t_{k}\right)=I_{k}\left(x\left(t_{k}^{-}\right)\right)+J_{k}\left(x\left(t_{k}^{-}-\tau\right)\right), \quad k \in \mathbb{Z}_{+} .
\end{gathered}
$$

However, their results are only valid for some specific systems due to the restrictive requirements on the continuous flows and impulsive gain. Lin et al. [36] investigated the exponential stability and uniform stability of the following more generalized IFDSs with delayed impulses:

$$
\begin{gathered}
\dot{x}(t)=f\left(t, x_{t}\right), \quad t \neq t_{k}, t \geqslant t_{0}, \\
\Delta x\left(t_{k}\right)=I_{k}\left(x\left(t_{k}^{-}\right)\right)+J_{k}\left(x_{t_{k}^{-}}\right), \quad k \in \mathbb{Z}_{+} .
\end{gathered}
$$

But those results can only been applied to the systems with stable discrete dynamics since their results need the strong condition of impulsive gain $d_{k}+e_{k}<1$.

Motivated by the above discussions, in this paper, we further study the exponential stability of IFDSs with delayed impulses. Different from the previous works on exponential stability of IFDSs with/without delayed impulses [18, 31, 34, 36], we will divide the systems into two classes: the system with stable continuous dynamics and unstable discrete dynamics, the systems with unstable continuous dynamics and stable discrete dynamics. The first class of impulsive systems corresponds to the case when the continuous dynamics are subjected to impulsive perturbations, while the second class of impulsive systems corresponds to the case when impulses are employed to stabilize the unstable continuous dynamics. This idea is enlightened in part by the works Chen and Zheng [37] about the uncertain impulsive systems. By using the Lyapunov functions and the Razumikhin techniques, some global exponential stability criteria are derived. The results obtained improve and complement some recent works. It is worth mentioning that our results shown that the system will be stable if the impulses' frequency and amplitude are suitably related to the increase or decrease of the continuous flows. Moreover, some results obtained can be applied to IFDSs with any time 
delay. In the end, an example is provided to illustrate the effectiveness and the advantages of the results obtained.

\section{Preliminaries}

Let $\mathbb{R}$ denote the set of real numbers, $\mathbb{R}_{+}$the set of nonnegative real numbers, $\mathbb{Z}_{+}$the set of positive integers, and $\mathbb{R}^{n}$ the $n$-dimensional real space equipped with the Euclidean norm $|\cdot|$. Let $\tau>0$ and $\mathrm{PC}\left([-\tau, 0] ; \mathbb{R}^{n}\right)=\left\{\varphi:[-\tau, 0] \rightarrow \mathbb{R}^{n} \mid \varphi\left(t^{+}\right)=\varphi(t)\right.$ for all $t \in[-\tau, 0)$, $\varphi\left(t^{-}\right)$exist and $\varphi\left(t^{-}\right)=\varphi(t)$ for all, but at most a finite number of points $\left.t \in(-\tau, 0]\right\}$ be with the norm $\|\varphi\|=\sup _{-\tau \leqslant \theta \leqslant 0}|\varphi(\theta)|$, where $\varphi\left(t^{+}\right)$and $\varphi\left(t^{-}\right)$denote the right-hand and left-hand limits of function $\varphi(t)$ at $t$, respectively. Denote $\mathrm{PC}\left(\left[t_{0}-\tau, b\right] ; \mathbb{R}_{+}\right)=\left\{\varphi:\left[t_{0}-\tau, b\right] \rightarrow \mathbb{R}_{+} \mid\right.$ $\varphi$ is piecewise continuous $\}$ for $b>t_{0}$, and $\operatorname{PC}\left(\left[t_{0}-\tau, \infty\right) ; \mathbb{R}_{+}\right)=\left\{\varphi|\varphi|_{\left[t_{0}-\tau, b\right]} \in \operatorname{PC}\left(\left[t_{0}-\tau, b\right] ; \mathbb{R}_{+}\right)\right.$ for all $\left.b>t_{0}-\tau\right\}$.

Consider the IFDS in which the state variables on the impulses are related to the time delay:

$$
\begin{gathered}
\dot{x}(t)=f\left(t, x_{t}\right), \quad t \neq t_{k}, t \geqslant t_{0}, \\
\Delta x\left(t_{k}\right)=I_{k}\left(t_{k}, x\left(t_{k}^{-}\right)\right)+J_{k}\left(t_{k}, x_{t_{k}^{-}}\right), \quad k \in \mathbb{Z}_{+} \\
x_{t_{0}}=\phi(s), \quad s \in[-\tau, 0],
\end{gathered}
$$

where $x \in \mathbb{R}^{n}, f: \mathbb{R}_{+} \times \mathbb{C} \rightarrow \mathbb{R}^{n}, I_{k}: \mathbb{R}_{+} \times \mathbb{R}^{n} \rightarrow \mathbb{R}^{n}, J_{k}: \mathbb{R}_{+} \times \mathbb{C} \rightarrow \mathbb{R}^{n}, \phi \in \mathrm{PC}\left([-\tau, 0] ; \mathbb{R}^{n}\right), \mathbb{C}$ is a open set in $\mathrm{PC}\left([-\tau, 0] ; \mathbb{R}^{n}\right)$. and The fixed moments of impulse times $\left\{t_{k}, k \in \mathbb{Z}_{+}\right\}$satisfy $0 \leqslant$ $t_{0}<t_{1}<\cdots<t_{k}<\cdots, t_{k} \rightarrow \infty($ as $k \rightarrow \infty), \Delta x\left(t_{k}\right)=x\left(t_{k}\right)-x\left(t_{k}^{-}\right) ; x_{t}, x_{t^{-}} \in \mathrm{PC}\left([-\tau, 0] ; \mathbb{R}^{n}\right)$ are defined by $x_{t}=x(t+\theta), x_{t^{-}}=x\left(t^{-}+\theta\right)$ for $\theta \in[-\tau, 0]$, respectively.

Throughout this paper, we assume that $f, I_{k}$, and $J_{k}, k \in \mathbb{Z}_{+}$, satisfy the necessary conditions for the global existence and uniqueness of solutions for all $t \geqslant t_{0}$, see $[6,30-$ $33]$. Then for any $\phi \in \mathrm{PC}\left([-\tau, 0] ; \mathbb{R}^{n}\right)$, there exists a unique function satisfying system (2.1) denoted by $x\left(t ; t_{0}, \phi\right)$, which is continuous on the right-hand side and limitable on the lefthand side. Moreover, we assume that $f(t, 0) \equiv 0, I_{k}\left(t_{k}, 0\right) \equiv 0$ and $J_{k}\left(t_{k}, 0\right) \equiv 0, k \in \mathbb{Z}_{+}$, which imply that $x(t) \equiv 0$ is a solution of (2.1), which is called the trivial solution.

At the end of this section, let us introduce the following definitions.

Definition 2.1. A function $V:\left[t_{0}-\tau, \infty\right) \times \mathbb{R}^{n} \rightarrow \mathbb{R}_{+}$belongs to class $v_{0}$ if

(i) $V$ is continuous on each of the sets $\left[t_{k-1}, t_{k}\right) \times \mathbb{R}^{n}$, and for each $x \in \mathbb{R}^{n}, t \in\left[t_{k-1}, t_{k}\right)$, $k \in \mathbb{Z}_{+}, \lim _{(t, y) \rightarrow\left(t_{k}^{-}, x\right)} V(t, y)=V\left(t_{k^{-}}^{-} x\right)$ exists;

(ii) $V(t, x)$ is locally Lipschitz in $x \in \mathbb{R}^{n}$, and $V(t, 0) \equiv 0$ for all $t \geqslant t_{0}$.

Definition 2.2. Given a function $V \in v_{0}$, the upper right-hand Dini derivative of $V$ with respect to system (2.1) is defined by

$$
D^{+} V(t, \psi(0))=\limsup _{h \rightarrow 0^{+}} \frac{1}{h}[V(t+h, \psi(0)+h f(t, \psi))-V(t, \psi(0))]
$$

for $(t, \psi) \in\left[t_{0}, \infty\right) \times \operatorname{PC}\left([-\tau, 0] ; \mathbb{R}^{n}\right)$. 
Definition 2.3. The trivial solution of system (2.1) or, simply, system (2.1) is said to be globally exponentially stable if there exist positive constants $\alpha$ and $C$ such that for any initial data $x_{t_{0}}=\phi \in \mathrm{PC}\left([-\tau, 0] ; \mathbb{R}^{n}\right)$, the solution $x\left(t ; t_{0}, \phi\right)$ satisfies

$$
\left|x\left(t ; t_{0}, \phi\right)\right| \leqslant C\|\phi\| e^{-\alpha\left(t-t_{0}\right)}, \quad t \geqslant t_{0}
$$

\section{Main Results}

In this section, we shall analyze the global exponential stability of system (2.1) by employing the Razumikhin techniques and the Lyapunov functions.

Theorem 3.1. Assume that there exist functions $V \in v_{0}, c \in \mathrm{PC}\left(\left[t_{0}-\tau, \infty\right) ; \mathbb{R}_{+}\right)$, several positive constants $c_{1}, c_{2}, \tilde{c}, p, q$, and nonnegative constants $\rho_{1}, \rho_{2}, \rho_{1}+\rho_{2} \geqslant 1$ such that

(i) $c_{1}|x|^{p} \leqslant V(t, x) \leqslant c_{2}|x|^{p}$, for all $(t, x) \in\left[t_{0}-\tau, \infty\right) \times \mathbb{R}^{n}$;

(ii) $V\left(t_{k}, \varphi(0)\right) \leqslant \rho_{1}\left(1+\mu_{k}\right) V\left(t_{k^{-}}^{-} \varphi(0)\right)+\rho_{2}\left(1+\mu_{k}\right) \sup _{\theta \in[-\tau, 0]} V\left(t_{k}^{-}+\theta, \varphi(\theta)\right)$, for each $k \in \mathbb{Z}_{+}$ and $\varphi \in P C\left([-\tau, 0] ; \mathbb{R}^{n}\right)$, where $\mu_{k}, k \in \mathbb{Z}_{+}$, are nonnegative constants with $\Sigma_{k=1}^{\infty} \mu_{k}<\infty$;

(iii) $D^{+} V(t, \varphi(0)) \leqslant-c(t) V(t, \varphi(0))$, for all $t \geqslant t_{0}, t \neq t_{k}, k \in \mathbb{Z}_{+}, \varphi \in \mathrm{PC}\left([-\tau, 0] ; \mathbb{R}^{n}\right)$, whenever $V(t+\theta, \varphi)<q V(t, \varphi(0)), \theta \in[-\tau, 0]$;

(iv) $\rho_{1}+\rho_{2} e^{\tilde{c} \tau}<q<e^{\tilde{c} \rho}, \inf _{t \geqslant t_{0}} c(t) \geqslant \tilde{c}$, where $\rho=\inf _{k \in \mathbb{Z}_{+}}\left\{t_{k}-t_{k-1}\right\}$.

Then the trivial solution of system (2.1) is globally exponentially stable and the convergence rate should not be greater than $(1 / p)(\widetilde{c}-(\ln q / Q))$.

Proof. Set $L=\prod_{k=1}^{\infty}\left(1+\mu_{k}\right)$; from the condition $\sum_{k=1}^{\infty} \mu_{k}<\infty$, we known that $1 \leqslant L<\infty$. Fix any initial data $\phi \in \mathrm{PC}\left([-\tau, 0] ; \mathbb{R}^{n}\right)$ and write $x\left(t ; t_{0}, \phi\right)=x(t), V(t, x(t))=V(t)$ simply. From condition (iv), we can choose a small enough constant $\gamma>0$ such that

$$
e^{\gamma \tau}\left(\rho_{1}+\rho_{2} e^{\tilde{c} \tau}\right)<q<e^{(\tilde{c}-\gamma) \rho}, \quad \gamma<\tilde{c}
$$

Set $\tilde{q}=q e^{-\gamma \tau}>1$, choose $M>0$ such that $\tilde{q} c_{2}<M$. Define $W(t)=e^{\gamma\left(t-t_{0}\right)} V(t)$. In the following, we shall show that

$$
W(t) \leqslant L M\|\phi\|^{p}, \quad t \geqslant t_{0} .
$$

In order to do so, we first prove that

$$
W(t)<M\|\phi\|^{p}, \quad t \in\left[t_{0}-\tau, t_{1}\right) .
$$

It is noted that

$$
W\left(t_{0}+\theta\right) \leqslant c_{2}\|\phi\|^{p}<\frac{1}{\tilde{q}} M\|\phi\|^{p}<M\|\phi\|^{p}, \quad \theta \in[-\tau, 0] .
$$


So it only needs to prove

$$
W(t)<M\|\phi\|^{p}, \quad t \in\left(t_{0}, t_{1}\right) .
$$

We assume, on the contrary, there exist some $t \in\left(t_{0}, t_{1}\right)$ such that $W(t) \geqslant M\|\phi\|^{p}$. Set

$$
t^{*}=\inf \left\{t \in\left[t_{0}, t_{1}\right): W(t) \geqslant M\|\phi\|^{p}\right\} .
$$

Note that $W(t)$ is continuous on $t \in\left[t_{0}, t_{1}\right)$, then $t^{*} \in\left(t_{0}, t_{1}\right)$ and

$$
W\left(t^{*}\right)=M\|\phi\|^{p}, \quad W(t)<M\|\phi\|^{p}, \quad t \in\left[t_{0}-\tau, t^{*}\right) .
$$

Define

$$
t^{* *}=\sup \left\{t \in\left[t_{0}, t^{*}\right]: W(t) \leqslant \frac{1}{\tilde{q}} M\|\phi\|^{p}\right\}
$$

then $t^{* *} \in\left(t_{0}, t^{*}\right)$ and

$$
W\left(t^{* *}\right)=\frac{1}{\tilde{q}} M\|\phi\|^{p}, \quad W(t)>\frac{1}{\tilde{q}} M\|\phi\|^{p}, \quad t \in\left(t^{* *}, t^{*}\right]
$$

Consequently, for all $t \in\left[t^{* *}, t^{*}\right]$,

$$
W(t+\theta) \leqslant M\|\phi\|^{p} \leqslant \tilde{q} W(t), \quad \theta \in[-\tau, 0]
$$

which implies that

$$
V(t+\theta)=e^{-\gamma\left(t+\theta-t_{0}\right)} W(t+\theta) \leqslant \tilde{q} e^{-\gamma\left(t+\theta-t_{0}\right)} W(t) \leqslant \tilde{q} e^{\gamma \tau} V(t)=q V(t), \quad \theta \in[-\tau, 0] .
$$

Then it follows from condition (iii) that one has that

$$
D^{+} W(t)=e^{\gamma\left(t-t_{0}\right)}\left[\gamma V(t)+D^{+} V(t)\right] \leqslant(\gamma-c(t)) W(t), \quad t \in\left[t^{* *}, t^{*}\right]
$$

which leads to

$$
\begin{gathered}
W\left(t^{*}\right) \leqslant W\left(t^{* *}\right) e^{\int_{t^{* *}}^{t^{*}}(\gamma-c(s)) \mathrm{d} s} \leqslant W\left(t^{* *}\right) e^{(\gamma-\widetilde{c})\left(t^{*}-t^{* *}\right)} \\
\leqslant \frac{1}{\tilde{q}} M\|\phi\|^{p}<M\|\phi\|^{p}
\end{gathered}
$$

this is a contradiction. Thus (3.5) holds. 
Now we assume that for some $m \in \mathbb{Z}_{+}, m \geqslant 1$,

$$
W(t)<M_{m}\|\phi\|^{p}, \quad t \in\left[t_{0}-\tau, t_{m}\right),
$$

where $M_{1}=M, M_{m}=M \prod_{1 \leqslant i \leqslant m-1}\left(1+\mu_{i}\right)$ for $m \geqslant 2$. We will prove that

$$
W(t)<M_{m+1}\|\phi\|^{p}, \quad t \in\left[t_{m}, t_{m+1}\right) .
$$

To do this, we first claim

$$
W\left(t_{m}^{-}+\theta\right) \leqslant \frac{e^{(\tilde{c}-\gamma) \tau}}{\tilde{q}} M_{m}\|\phi\|^{p}, \quad \theta \in[-\tau, 0) .
$$

Suppose not, then there exists $\tilde{\theta} \in[-\tau, 0)$ such that $W\left(t_{m}^{-}+\tilde{\theta}\right)>\left(e^{(\tilde{c}-\gamma) \tau} / \tilde{q}\right) M_{m}\|\phi\|^{p}$. Without lose generality, we assume $t_{m}+\tilde{\theta} \in\left(t_{l-1}, t_{l}\right], l \in \mathbb{Z}_{+}, l \leqslant m$.

There are two cases to be considered.

Case 1. $W(t)>\left(e^{(\tilde{c}-\gamma) \tau} / \tilde{q}\right) M_{m}\|\phi\|^{p}$ over $t \in\left[t_{l-1}, t_{m}+\tilde{\theta}\right)$.

By assumption (3.14), for all $t \in\left[t_{l-1}, t_{m}+\tilde{\theta}\right)$, we get

$$
W(t+\theta)<M_{m}\|\phi\|^{p}<e^{(\widetilde{c}-\gamma) \tau} M_{m}\|\phi\|^{p}<\tilde{q} W(t), \quad \theta \in[-\tau, 0] .
$$

Thus, by conditions (iii)-(iv) and inequalities (3.10)-(3.13), we have

$$
\begin{aligned}
W\left(t_{m}^{-}+\tilde{\theta}\right) & \leqslant W\left(t_{l-1}\right) e^{(\gamma-\widetilde{c})\left(t_{m}^{-}+\tilde{\theta}-t_{l-1}\right)} \\
& <M_{m}\|\phi\|^{p} e^{(\tilde{c}-\gamma) \tau} e^{(\gamma-\tilde{c})\left(t_{m}-t_{l-1}\right)} \\
& \leqslant \frac{e^{(\widetilde{c}-\gamma) \tau}}{q^{m-l+1}} M_{m}\|\phi\|^{p} \\
& <\frac{e^{(\widetilde{c}-\gamma) \tau}}{\tilde{q}} M_{m}\|\phi\|^{p}
\end{aligned}
$$

This is a contradiction.

Case 2. There are some $t \in\left[t_{l-1}, t_{m}+\tilde{\theta}\right)$ such that $W(t)>\left(e^{(\tilde{c}-\gamma) \tau} / \tilde{q}\right) M_{m}\|\phi\|^{p}$.

In this case, define

$$
\bar{t}=\sup \left\{t \in\left[t_{l-1}, t_{m}+\tilde{\theta}\right): W(t) \leqslant \frac{e^{(\tilde{c}-\gamma) \tau}}{\tilde{q}} M_{m}\|\phi\|^{p}\right\}
$$

Then $\bar{t} \in\left[t_{l-1}, t_{m}+\tilde{\theta}\right)$ and

$$
W(\bar{t})=\frac{e^{(\tilde{c}-\gamma) \tau}}{\tilde{q}} M_{m}\|\phi\|^{p}, \quad W(t)>\frac{e^{(\tilde{c}-\gamma) \tau}}{\tilde{q}} M_{m}\|\phi\|^{p}, \quad t \in\left(\bar{t}, t_{m}+\tilde{\theta}\right) .
$$


So from assumption (3.14), for any $t \in\left[\bar{t}, t_{m}+\tilde{\theta}\right)$, we have

$$
W(t+\theta)<M_{m}\|\phi\|^{p}<e^{(\widetilde{c}-\gamma) \tau} M_{m}\|\phi\|^{p} \leqslant \tilde{q} W(t), \quad \theta \in[-\tau, 0] .
$$

It follows from condition (iii) that

$$
W\left(t_{m}^{-}+\tilde{\theta}\right) \leqslant W(\bar{t})=\frac{e^{(\tilde{c}-\gamma) \tau}}{\tilde{q}} M_{m}\|\phi\|^{p}
$$

This is also a contradiction. Hence, inequality (3.16) holds.

Similarly, we can prove

$$
W\left(t_{m}^{-}\right) \leqslant \frac{1}{\tilde{q}} M_{m}\|\phi\|^{p} .
$$

Then it follows from (3.16), (3.23), and condition (ii) that we obtain

$$
\begin{aligned}
W\left(t_{m}\right) & \leqslant \rho_{1}\left(1+\mu_{m}\right) W\left(t_{m}^{-}\right)+\rho_{2}\left(1+\mu_{m}\right) e^{\gamma \tau} \sup _{\theta \in[-\tau, 0]} W\left(t_{m}^{-}+\theta\right) \\
& \leqslant \frac{\rho_{1}+\rho_{2} e^{\tilde{\mathcal{c}} \tau}}{\tilde{q}} M_{m+1}\|\phi\|^{p}<M_{m+1}\|\phi\|^{p} .
\end{aligned}
$$

Now we suppose that (3.15) is not true, let

$$
t^{*}=\inf \left\{t \in\left[t_{m}, t_{m+1}\right): W(t) \geqslant M_{m+1}\|\phi\|^{p}\right\} .
$$

Then $t^{*} \in\left(t_{m}, t_{m+1}\right)$ and

$$
W\left(t^{*}\right)=M_{m+1}\|\phi\|^{p}, \quad W(t)<M_{m+1}\|\phi\|^{p}, \quad t \in\left[t_{m}, t^{*}\right) .
$$

If $W(t)>(1 / \widetilde{q}) M_{m+1}\|\phi\|^{p}$ for all $t \in\left[t_{m}, t^{*}\right]$, set $t^{* *}=t_{m}$; otherwise, let

$$
t^{* *}=\sup \left\{t \in\left[t_{m}, t^{*}\right]: W(t) \leqslant \frac{1}{\tilde{q}} M_{m+1}\|\phi\|^{p}\right\}
$$

Thus for all $t \in\left[t^{* *}, t^{*}\right]$, we have

$$
W(t+\theta) \leqslant M_{m+1}\|\phi\|^{p} \leqslant \tilde{q} W(t), \quad \theta \in[-\tau, 0] .
$$

It follows from condition (iii) that

$$
D^{+} W(t)=e^{\gamma\left(t-t_{0}\right)}\left[\gamma V(t)+D^{+} V(t)\right] \leqslant(\gamma-c(t)) W(t), \quad t \in\left[t^{* *}, t^{*}\right],
$$


which implies

$$
W\left(t^{*}\right) \leqslant W\left(t^{* *}\right) e^{(\gamma-\widetilde{c})\left(t^{*}-t^{* *}\right)} \leqslant W\left(t^{* *}\right)<M_{m+1}\|\phi\|^{p} .
$$

This is a contradiction. Therefore, (3.15) holds.

By mathematical induction, (3.15) holds for any $m \in \mathbb{Z}_{+}$. That is, (3.2) holds, which implies that

$$
|x(t)| \leqslant C\|\phi\| e^{(-\gamma / p)\left(t-t_{0}\right)}, \quad t \geqslant t_{0}
$$

where $C=\left(\mathrm{LM} / c_{1}\right)^{1 / p}$. This completes the proof.

Remark 3.2. The parameters $\rho_{1}$ and $\rho_{2}$ in condition (ii) describe the influence of impulses on the stability of the underlying continuous systems. When $\rho_{1}+\rho_{2} \geqslant 1$, the Lyapunov function $V$ may jump up along the state trajectories of system (2.1) at impulsive time instant $t_{k}$. Thus the impulses may be viewed as disturbances, that is, they potentially destroy the stability of continuous system. In this case, it is required that the impulses do not occur too frequently. Theorem 3.1 tells us to what extent we can relax the restriction on the impulses to keep the exponential stability property of the original continuous system.

Theorem 3.3. Assume that there exist functions $V \in v_{0}, c \in \mathrm{PC}\left(\left[t_{0}-\tau, \infty\right) ; \mathbb{R}_{+}\right)$, several positive constants $c_{1}, c_{2}, \tilde{c}, p, q$, and nonnegative constants $\rho_{1}, \rho_{2}, \rho_{1}+\rho_{2}<1$ such that

(i) $c_{1}|x|^{p} \leqslant V(t, x) \leqslant c_{2}|x|^{p}$, for all $(x, t) \in \mathbb{R}^{n} \times\left[t_{0}-\tau, \infty\right)$;

(ii) $V\left(t_{k}, \varphi(0)\right) \leqslant \rho_{1}\left(1+\mu_{k}\right) V\left(t_{k}^{-}, \varphi(0)\right)+\rho_{2}\left(1+\mu_{k}\right) \sup _{\theta \in[-\tau, 0]} V\left(t_{k}^{-}+\theta, \varphi(\theta)\right)$, for each $k \in \mathbb{Z}_{+}$ and $\varphi \in P\left([-\tau, 0] ; \mathbb{R}^{n}\right)$, where $\mu_{k}, k \in \mathbb{Z}_{+}$, are nonnegative constants with $\sum_{k=1}^{\infty} \mu_{k}<\infty$;

(iii) $D^{+} V(t, \varphi(0)) \leqslant c(t) V(t, \varphi(0))$, for all $t \geqslant t_{0}, t \neq t_{k}, k \in \mathbb{Z}_{+}, \varphi \in \mathrm{PC}\left([-\tau, 0] ; \mathbb{R}^{n}\right)$, whenever $V(t+\theta, \varphi)<q V(t, \varphi(0)), \theta \in[-\tau, 0]$;

(iv) $q>1 /\left(\rho_{1}+\rho_{2}\right)>e^{\tilde{c} \rho}, \tilde{c} \rho \geqslant \sup _{t \geqslant t_{0}} \int_{t}^{t+\rho} c(s) d s$, where $\rho=\sup _{k \in \mathbb{Z}_{+}}\left\{t_{k}-t_{k-1}\right\}$.

Then the trivial solution of system (2.1) is globally exponentially stable for any time delay $\tau \in(0, \infty)$ and the convergence rate should not be greater than $(1 / p)((\ln q / \rho)-\widetilde{c})$.

Proof. From condition (iv), we can choose a small enough constant $\gamma>0$ such that

$$
q>\frac{e^{\gamma \tau}}{\rho_{1}+\rho_{2} e^{\gamma \tau}}>\frac{1}{\rho_{1}+\rho_{2} e^{\gamma \tau}}>e^{(\tilde{c}+\gamma) \rho}, \quad q e^{-\gamma \tau}>1
$$

Set $\tilde{q}=q e^{-\gamma \tau}$. The following proof can be completed by using the similar arguments as in the proof of Theorem 3.1, so it is omitted.

Remark 3.4. When $\rho_{1}+\rho_{2}<1$, the Lyapunov function $V$ may jump down along the state trajectories of system (2.1) at impulsive time instant $t_{k}$. Thus the impulses may be viewed impulsive stabilizing, that is, they may be used to stabilize the continuous system if the original continuous system is not stable. In this case, the impulses must be frequent and their amplitude must be suitably related the growth rate of $V$. 
Remark 3.5. If $c(t) \equiv c$, then Theorem 3.3 becomes Theorem 3.1 in [36] with $d_{k}=\rho_{1}\left(1+\mu_{k}\right)$, $e_{k}=\rho_{2}\left(1+\mu_{k}\right), d_{k}+e_{k}<1$. Obviously, Theorem 3.3 in this paper has a wider adaptive range than those in [36].

Let $J_{k} \equiv 0$ in system (2.1), then we have the following IFDS (see [9-23, 26]):

$$
\begin{gathered}
\dot{x}(t)=f\left(x_{t}, t\right), \quad t \neq t_{k}, \quad t \geqslant t_{0}, \\
\Delta x\left(t_{k}\right)=I_{k}\left(x\left(t_{k}^{-}\right), t_{k}\right), \quad k \in \mathbb{Z}_{+}, \\
x_{t_{0}}=\phi(s), \quad s \in[-\tau, 0] .
\end{gathered}
$$

For system (3.33), we have the following results by Theorems 3.1 and 3.3, respectively.

Corollary 3.6. Assume that there exist functions $V \in v_{0}, c \in \operatorname{PC}\left(\left[t_{0}-\tau, \infty\right) ; \mathbb{R}_{+}\right)$, and several positive constants $c_{1}, c_{2}, \tilde{c}, p, q$, and a constant $\rho \geqslant 1$ such that

(i) $c_{1}|x|^{p} \leqslant V(t, x) \leqslant c_{2}|x|^{p}$, for all $(x, t) \in \mathbb{R}^{n} \times\left[t_{0}-\tau, \infty\right)$;

(ii) $V\left(t_{k}, \varphi(0)\right) \leqslant \rho\left(1+\mu_{k}\right) V\left(t_{k}^{-}, \varphi(0)\right)$, for each $k \in \mathbb{Z}_{+}$and $\varphi \in P\left([-\tau, 0] ; \mathbb{R}^{n}\right)$, where $\mu_{k}$, $k \in \mathbb{Z}_{+}$, are nonnegative constants with $\sum_{k=1}^{\infty} \mu_{k}<\infty$;

(iii) $D^{+} V(t, \varphi(0)) \leqslant-c(t) V(t, \varphi(0))$, for all $t \geqslant t_{0}, t \neq t_{k}, k \in \mathbb{Z}_{+}, \varphi \in \mathrm{PC}\left([-\tau, 0] ; \mathbb{R}^{n}\right)$, whenever $V(t+\theta, \varphi)<q V(t, \varphi(0)), \theta \in[-\tau, 0]$;

(iv) $\rho<q<e^{\tilde{c} \rho}, \inf _{t \geqslant t_{0}} c(t) \geqslant \tilde{c}$, where $\rho=\inf _{k \in \mathbb{Z}_{+}}\left\{t_{k}-t_{k-1}\right\}$.

Then the trivial solution of system (3.33) is globally exponentially stable for any time delay $\tau \in(0, \infty)$ and the convergence rate should not be greater than $(1 / p)(\tilde{c}-(\ln q / \rho))$.

Corollary 3.7. Assume that there exist functions $V \in v_{0}, c \in \mathrm{PC}\left(\left[t_{0}-\tau, \infty\right) ; \mathbb{R}_{+}\right)$and several positive constants $c_{1}, c_{2}, \widetilde{c}, p, q$, and a constant $\rho<1$ such that

(i) $c_{1}|x|^{p} \leqslant V(t, x) \leqslant c_{2}|x|^{p}$, for all $(x, t) \in \mathbb{R}^{n} \times\left[t_{0}-\tau, \infty\right)$;

(ii) $V\left(t_{k}, \varphi(0)\right) \leqslant \rho\left(1+\mu_{k}\right) V\left(t_{k}^{-}, \varphi(0)\right)$, for each $k \in \mathbb{Z}_{+}, \varphi \in P\left([-\tau, 0] ; \mathbb{R}^{n}\right)$, where $\mu_{k}$, $k \in \mathbb{Z}_{+}$, are nonnegative constants with $\sum_{k=1}^{\infty} \mu_{k}<\infty$;

(iii) $D^{+} V(t, \varphi(0)) \leqslant c(t) V(t, \varphi(0))$, for all $t \geqslant t_{0}, t \neq t_{k}, k \in \mathbb{Z}_{+}, \varphi \in \mathrm{PC}\left([-\tau, 0] ; \mathbb{R}^{n}\right)$, whenever $V(t+\theta, \varphi)<q V(t, \varphi(0)), \theta \in[-\tau, 0]$;

(iv) $q>1 / \rho>e^{\tilde{c} \rho}, \tilde{c} \rho \geqslant \sup _{t \geqslant t_{0}} \int_{t}^{t+\rho} c(s) d s$, where $\rho=\sup _{k \in \mathbb{Z}_{+}}\left\{t_{k}-t_{k-1}\right\}$.

Then the trivial solution of system (3.33) is globally exponentially stable for any time delay $\tau \in(0, \infty)$ and the convergence rate should not be greater than $(1 / p)((\ln q / Q)-\widetilde{c})$.

Remark 3.8. If $c(t) \equiv c>0, \mu_{k} \equiv 0, k \in \mathbb{Z}_{+}$, then Theorems 3.1 and 3.2 in [25] follow from Corollaries 3.6 and 3.7, respectively.

\section{Example}

In this section, an example is given to show the effectiveness and advantages of our results. 
Example 4.1. Consider the following IFDS (see $[35,36])$ :

$$
\begin{aligned}
& \dot{x}(t)=a x(t)+b x(t-\tau), \quad t \neq t_{k}, \quad t>0, \\
& x\left(t_{k}\right)=c x\left(t_{k}^{-}\right)+d x\left(t_{k}^{-}-\tau\right), \quad k \in \mathbb{Z}_{+},
\end{aligned}
$$

where $x \in \mathbb{R}, \tau>0$.

In the following, we will divide the system (4.1) into two classes to consider.

Case 1. $a \geqslant 0$ and $0<|c|+|d|<1$.

Property 1. The trivial solution of system (4.1) is globally exponentially stable with impulse time sequences that satisfy

$$
\sup _{k \in \mathbb{Z}_{+}}\left\{t_{k}-t_{k-1}\right\}<-\frac{(|c|+|d|) \ln (|c|+|d|)}{a(|c|+|d|)+|b|}
$$

Proof. From equality (4.2), one can choose a small enough constant $h>0$ such that

$$
\begin{gathered}
|c|+|d|-h>0 \\
\sup _{k \in \mathbb{Z}_{+}}\left\{t_{k}-t_{k-1}\right\}<-\frac{(|c|+|d|-h) \ln (|c|+|d|)}{a(|c|+|d|-h)+|b|} .
\end{gathered}
$$

Let $V(t, x)=|x|$. By calculation, we have

$$
D^{+} V(t, \varphi(0)) \leqslant a|\varphi(0)|+|b||\varphi(-\tau)|=a V(t, \varphi(0))+|b| V(t, \varphi),
$$

for all $t \neq t_{k}, k \in \mathbb{Z}_{+}$and $\varphi \in \operatorname{PC}([-\tau, 0] ; \mathbb{R})$. By taking $p=1, c_{1}=c_{2}=1, \rho_{1}=|c|, \rho_{2}=|d|$, $q=1 /(|c|+|d|-h), \tilde{c}=c(t) \equiv a+(|b| /(|c|+|d|-h))$, and $\mu_{k} \equiv 0, k \in \mathbb{Z}_{+}$in Theorem 3.3, it is easy to obtain Property 1 .

Remark 4.2. In this case, the impulses are used to stabilize the unstable original continuous system. In [35], under assumption that $a, b, c, d>0$, and $c+d<1$, Zhang and Sun obtained that system (4.1) is uniformly stable if the impulses' instances satisfy

$$
\sup _{k \in \mathbb{Z}_{+}}\left\{t_{k}-t_{k-1}\right\}<\frac{-2(c+d)^{2} \ln (c+d)}{(2 a+b)(c+d)^{2}+b}
$$

Lin et al. [36] derived that system (4.1) is exponentially stable if

$$
\sup _{k \in \mathbb{Z}_{+}}\left\{t_{k}-t_{k-1}\right\}<-\frac{1}{2} \frac{(c+d) \ln (c+d)}{a(c+d)+b}
$$


Obviously, under condition $a, b, c, d>0$, and $c+d<1$, we get

$$
-\frac{(|c|+|d|) \ln (|c|+|d|)}{a(|c|+|d|)+|b|}=-\frac{(c+d) \ln (c+d)}{a(c+d)+b}>-\frac{1}{2} \frac{(c+d) \ln (c+d)}{a(c+d)+b},
$$

and one can also verify that

$$
-\frac{(c+d) \ln (c+d)}{a(c+d)+b}>\frac{-2(c+d)^{2} \ln (c+d)}{(2 a+b)(c+d)^{2}+b} .
$$

So our results are less conservative than those in $[35,36]$.

Case 2. $a<0$ and $|c|+|d| \geqslant 1$.

Property 2. Suppose that system's parameters $a, b, c, d$ and time delay $\tau$ satisfy

$$
(|c|+|d|)^{2} e^{-2 a \tau}<-\frac{2 a+|b|}{|b|} .
$$

Then the trivial solution of system (4.1) is globally exponentially stable with impulse time sequences that satisfy

$$
\inf _{k \in \mathbb{Z}_{+}}\left\{t_{k}-t_{k-1}\right\}>\frac{2 a \tau-2 \ln (|c|+|d|)}{2 a+|b|+|b|(|c|+|d|)^{2} e^{-2 a \tau}}
$$

Proof. From equalities (4.9) and (4.10), we can choose a small enough constant $h>0$ such that

$$
\begin{gathered}
(|c|+|d|)^{2} e^{-2 a \tau}<(|c|+|d|)^{2} e^{-2 a \tau}+h<-\frac{2 a+|b|}{|b|}, \\
\inf _{k \in \mathbb{Z}_{+}}\left\{t_{k}-t_{k-1}\right\}>-\frac{\ln \left[(|c|+|d|)^{2} e^{-2 a \tau}+2 h\right]}{2 a+|b|+|b|(|c|+|d|)^{2} e^{-2 a \tau}+h} .
\end{gathered}
$$

Set $q=(|c|+|d|)^{2} e^{-2 a \tau}+h$, then one can conclude that

$$
2 a+|b|+q|b|<0, \quad(|c|+|d|)^{2} e^{-(2 a+|b|+q|b|) \tau}<q<-\frac{2 a+|b|}{|b|} .
$$

Let $V(t, x)=(1 / 2) x^{2}$. By calculation, we have

$$
D^{+} V(t, \varphi(0)) \leqslant\left(a+\frac{1}{2}|b|\right) \varphi^{2}(0)+\frac{1}{2}|b| \varphi^{2}(-\tau)=(2 a+|b|) V(t, \varphi(0))+|b| V(t, \varphi),
$$

for all $t \neq t_{k}, k \in \mathbb{Z}_{+}$, and $\varphi \in \operatorname{PC}([-\tau, 0] ; \mathbb{R})$. By taking $p=2, c_{1}=c_{2}=2, \rho_{1}=|c|(|c|+|d|)$, $\rho_{2}=|d|(|c|+|d|), \tilde{c}=c(t) \equiv-(2 a+|b|+q|b|)$, and $\mu_{k} \equiv 0, k \in \mathbb{Z}_{+}$in Theorem 3.3, we can obtain Property 2. 
Remark 4.3. In this case, the underlying continuous system is stable, the impulses are disturbances, which potentially destroy the stability of continuous system. So the existing results in $[35,36]$ are invalid for this case.

\section{Conclusions}

This paper has studied the exponential stability of IFDSs in which the state variables on the impulses are related to the time delay. By using the Razumikhin techniques and the Lyapunov functions, some criteria on the global exponential stability are established. The obtained results improve and complement some recent works. An example has been given to illustrate the effectiveness and the advantages of the results obtained.

\section{Acknowledgments}

This work was supported by the 211 Project of the Anhui University (32030018/ 33010205/KJTD002B), the Research Fund for Doctor Station of the Ministry of Education of China (20113401110001), the Key Natural Science Foundation (KJ2009A49), the Foundation of Anhui Education Bureau (KJ2012A019), and the National Natural Science Foundation of China (11126179).

\section{References}

[1] V. Lakshmikantham, D. D. Baĭnov, and P. S. Simeonov, Theory of Impulsive Differential Equations, vol. 6, World Scientific Publishing, Singapore, 1989.

[2] W. M. Haddad, V. Chellaboina, and S. G. Nersesov, Impulsive and Hybrid Dynamical Systems: Stability, Dissipativity, and Control, Princeton University Press, Princeton, NJ, USA, 2006.

[3] K. Gopalsamy and B. G. Zhang, "On delay differential equations with impulses," Journal of Mathematical Analysis and Applications, vol. 139, no. 1, pp. 110-122, 1989.

[4] J. Shen and X. Liu, "Global existence results for impulsive differential equations," Journal of Mathematical Analysis and Applications, vol. 314, no. 2, pp. 546-557, 2006.

[5] G. Ballinger and X. Liu, "Existence and uniqueness results for impulsive delay differential equations," Dynamics of Continuous, Discrete and Impulsive Systems, vol. 5, no. 1-4, pp. 579-591, 1999.

[6] G. Ballinger and X. Liu, "Existence, uniqueness and boundedness results for impulsive delay differential equations," Applicable Analysis, vol. 74, no. 1-2, pp. 71-93, 2000.

[7] A. Ouahab, "Existence and uniqueness results for impulsive functional differential equations with scalar multiple delay and infinite delay," Nonlinear Analysis: Theory, Methods E Applications, vol. 67, no. 4, pp. 1027-1041, 2007.

[8] M. Perestyuk and P. Feketa, "Invariant sets of impulsive differential equations with particularities in w-limit set," Abstract and Applied Analysis, vol. 2011, Article ID 970469, 14 pages, 2011.

[9] Z. Luo and J. Shen, "Stability and boundedness for impulsive functional differential equations with infinite delays," Nonlinear Analysis: Theory, Methods E Applications, vol. 46, no. 4, pp. 475-493, 2001.

[10] I. M. Stamova and G. T. Stamov, "Lyapunov-Razumikhin method for impulsive functional differential equations and applications to the population dynamics," Journal of Computational and Applied Mathematics, vol. 130, no. 1-2, pp. 163-171, 2001.

[11] Z. Luo and J. Shen, "New Razumikhin type theorems for impulsive functional differential equations," Applied Mathematics and Computation, vol. 125, no. 2-3, pp. 375-386, 2002.

[12] I. M. Stamova, "Vector Lyapunov functions for practical stability of nonlinear impulsive functional differential equations," Journal of Mathematical Analysis and Applications, vol. 325, no. 1, pp. 612-623, 2007.

[13] Z. Chen and X. Fu, "New Razumikhin-type theorems on the stability for impulsive functional differential systems," Nonlinear Analysis: Theory, Methods \& Applications, vol. 66, no. 9, pp. 2040-2052, 2007. 
[14] X. Liu and Q. Wang, "On stability in terms of two measures for impulsive systems of functional differential equations," Journal of Mathematical Analysis and Applications, vol. 326, no. 1, pp. 252-265, 2007.

[15] X. Fu and X. Li, "Razumikhin-type theorems on exponential stability of impulsive infinite delay differential systems," Journal of Computational and Applied Mathematics, vol. 224, no. 1, pp. 1-10, 2009.

[16] X. Li, "Uniform asymptotic stability and global stabiliy of impulsive infinite delay differential equations," Nonlinear Analysis: Theory, Methods \& Applications, vol. 70, no. 5, pp. 1975-1983, 2009.

[17] X. Li, "New results on global exponential stabilization of impulsive functional differential equations with infinite delays or finite delays," Nonlinear Analysis: Real World Applications, vol. 11, no. 5, pp. 4194-4201, 2010.

[18] X. Li, "Further analysis on uniform stability of impulsive infinite delay differential equations," Applied Mathematics Letters, vol. 25, no. 2, pp. 133-137, 2012.

[19] J. H. Shen, "Razumikhin techniques in impulsive functional-differential equations," Nonlinear Analysis: Theory, Methods \& Applications, vol. 36, no. 1, pp. 119-130, 1999.

[20] Z. Luo and J. Shen, "Impulsive stabilization of functional differential equations with infinite delays," Applied Mathematics Letters, vol. 16, no. 5, pp. 695-701, 2003.

[21] Y. Zhang and J. Sun, "Strict stability of impulsive functional differential equations," Journal of Mathematical Analysis and Applications, vol. 301, no. 1, pp. 237-248, 2005.

[22] Y. Zhang and J. Sun, "Stability of impulsive infinite delay differential equations," Applied Mathematics Letters, vol. 19, no. 10, pp. 1100-1106, 2006.

[23] A. Weng and J. Sun, "Impulsive stabilization of second-order delay differential equations," Nonlinear Analysis: Real World Applications, vol. 8, no. 5, pp. 1410-1420, 2007.

[24] A. Weng and J. Sun, "Impulsive stabilization of second-order nonlinear delay differential systems," Applied Mathematics and Computation, vol. 214, no. 1, pp. 95-101, 2009.

[25] S. Peng and L. Yang, "Global exponential stability of impulsive functional differential equations via Razumikhin technique," Abstract and Applied Analysis, vol. 2010, Article ID 987372, 11 pages, 2010.

[26] Z. Luo and J. Shen, "Stability of impulsive functional differential equations via the Liapunov functional," Applied Mathematics Letters, vol. 22, no. 2, pp. 163-169, 2009.

[27] F. Lian, J. Moyne, and D. Tilbury, "Modelling and optimal controller design of networked control systems with multiple delays," International Journal of Control, vol. 76, no. 6, pp. 591-606, 2003.

[28] A. Khadra, X. Z. Liu, and X. Shen, "Analyzing the robustness of impulsive synchronization coupled by linear delayed impulses," IEEE Transactions on Automatic Control, vol. 54, no. 4, pp. 923-928, 2009.

[29] X. Liu and G. Ballinger, "Uniform asymptotic stability of impulsive delay differential equations," Computers \& Mathematics with Applications, vol. 41, no. 7-8, pp. 903-915, 2001.

[30] X. Liu and G. Ballinger, "Boundedness for impulsive delay differential equations and applications to population growth models," Nonlinear Analysis: Theory, Methods E Applications, vol. 53, no. 7-8, pp. 1041-1062, 2003.

[31] Q. Wang and X. Liu, "Exponential stability for impulsive delay differential equations by Razumikhin method," Journal of Mathematical Analysis and Applications, vol. 309, no. 2, pp. 462-473, 2005.

[32] Q. Wang and X. Liu, "Impulsive stabilization of delay differential systems via the LyapunovRazumikhin method," Applied Mathematics Letters, vol. 20, no. 8, pp. 839-845, 2007.

[33] X. Liu and Q. Wang, "The method of Lyapunov functionals and exponential stability of impulsive systems with time delay," Nonlinear Analysis: Theory, Methods E Applications, vol. 66, no. 7, pp. 14651484, 2007.

[34] Q. Wu, J. Zhou, and L. Xiang, “Global exponential stability of impulsive differential equations with any time delays," Applied Mathematics Letters, vol. 23, no. 2, pp. 143-147, 2010.

[35] Y. Zhang and J. Sun, "Stability of impulsive functional differential equations," Nonlinear Analysis: Theory, Methods \& Applications, vol. 68, no. 12, pp. 3665-3678, 2008.

[36] D. Lin, X. Li, and D. O'Regan, "Stability analysis of generalized impulsive functional differential equations," Mathematical and Computer Modelling, vol. 55, no. 5-6, pp. 1682-1690, 2012.

[37] W. Chen and W. Zheng, "Robust stability and $H_{\infty}$-control of uncertain impulsive systems with timedelay," Automatica, vol. 45, no. 1, pp. 109-117, 2009. 


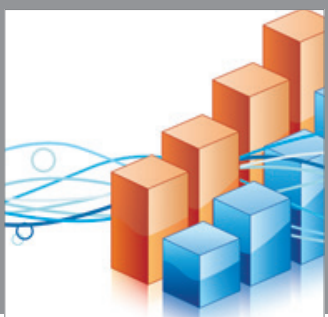

Advances in

Operations Research

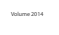

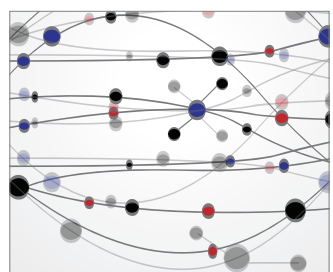

\section{The Scientific} World Journal
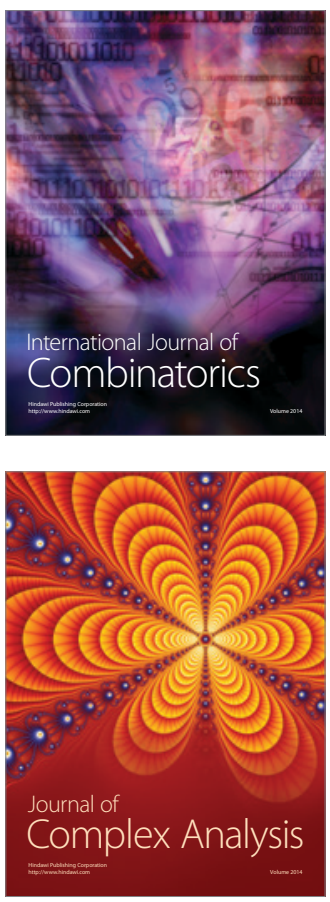

International Journal of

Mathematics and

Mathematical

Sciences
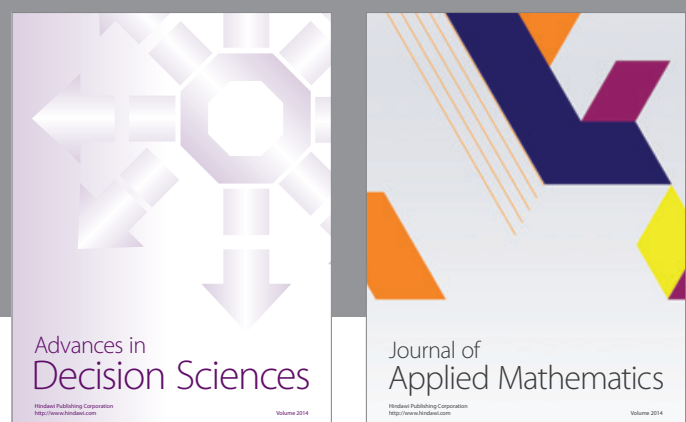

Journal of

Applied Mathematics
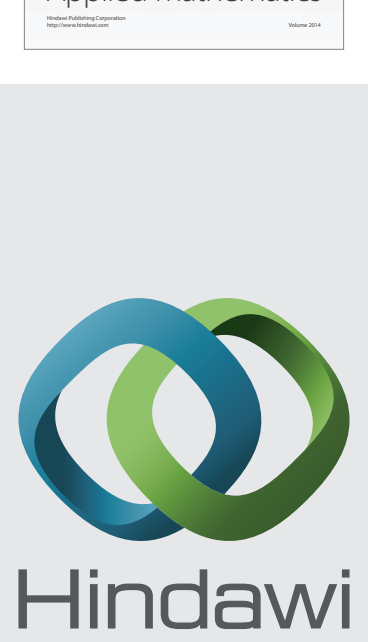

Submit your manuscripts at http://www.hindawi.com
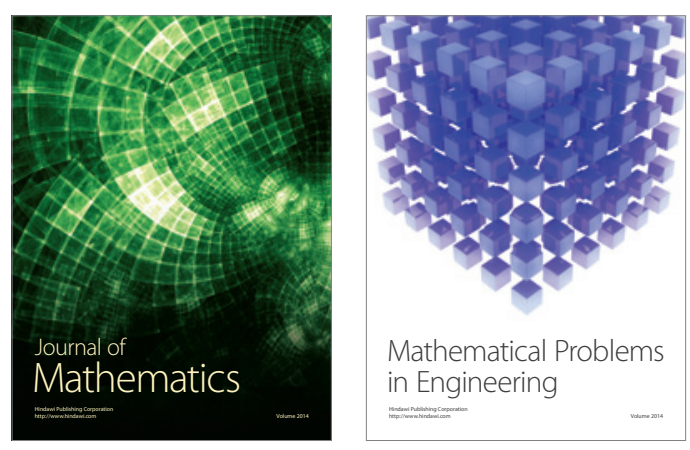

Mathematical Problems in Engineering
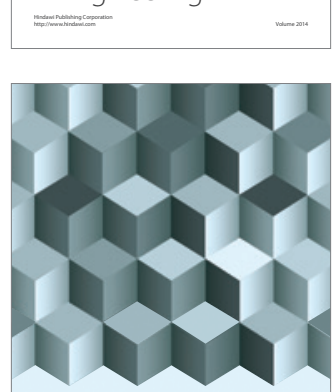

Journal of

Function Spaces
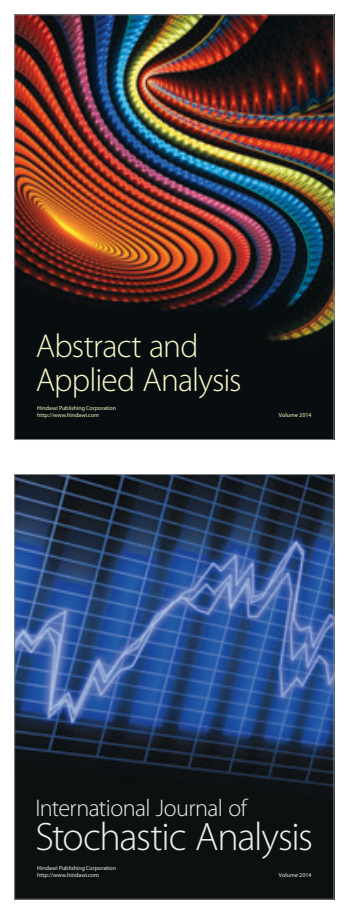

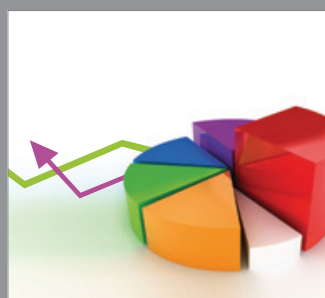

ournal of

Probability and Statistics

Promensencen
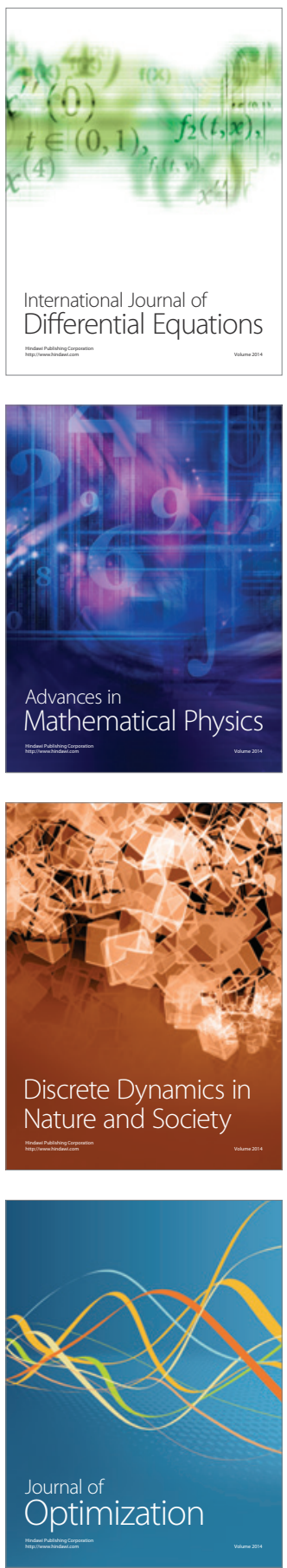